\title{
Statistics of chemical tracer concentration in a multi-compartment structure measured with a sensor network
}

\author{
$\underline{\text { Shanika Karunasekera }}^{\mathrm{a}}$, Alex Skvortsov ${ }^{\mathrm{b}}$, Ajith Gunatilaka $^{\mathrm{b}}$, and Dinesh Pitaliadda ${ }^{\mathrm{b}}$ \\ ${ }^{a}$ Department of Computing and Information Systems, University of Melbourne, VIC 3010 \\ ${ }^{\mathrm{b}}$ Land Division, DSTO, 506 Lorimer Street, Fishermans Bend, VIC 3207 \\ Email: karus@unimelb.edu.au
}

\begin{abstract}
An understanding of the statistics of concentrations of chemical tracers dispersing through multicompartment structures such as buildings, ships, and aircraft is important in many applications (e.g., industrial, military, and ecological). Such an understanding can be useful for early detection of hazardous releases, source backtracking, and assessment of their impact on engineered structures (e.g., buildings, aircraft, ships). It may be also important for design of monitoring sensor systems. We present the statistics of chemical tracer concentrations obtained by analysing recent experimental data obtained in a study of tracer dispersion in a complex structure. The experiment involved the release of a tracer (dyed salt solution) from a point source placed inside a multi-compartment structure (Fig. 1) embedded in a water tank and measuring the salt concentration at different locations within the compartments using a network of conductivity sensors. We show that moments of measured concentration at a given sensor depend on the Euclidean distance between the source and the sensor, resembling the behaviour observed in advection-diffusion transport in porous media.
\end{abstract}
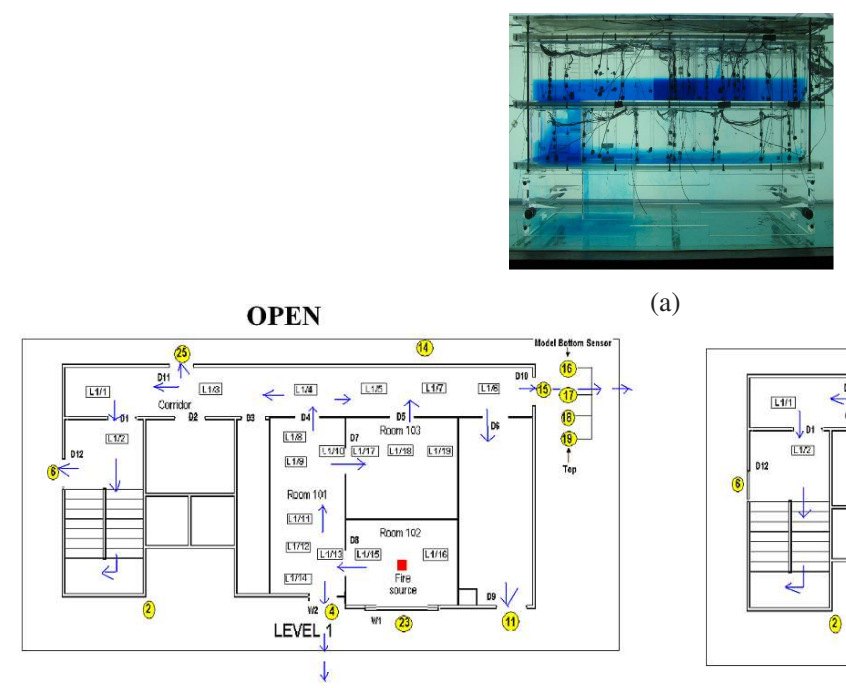

(a)
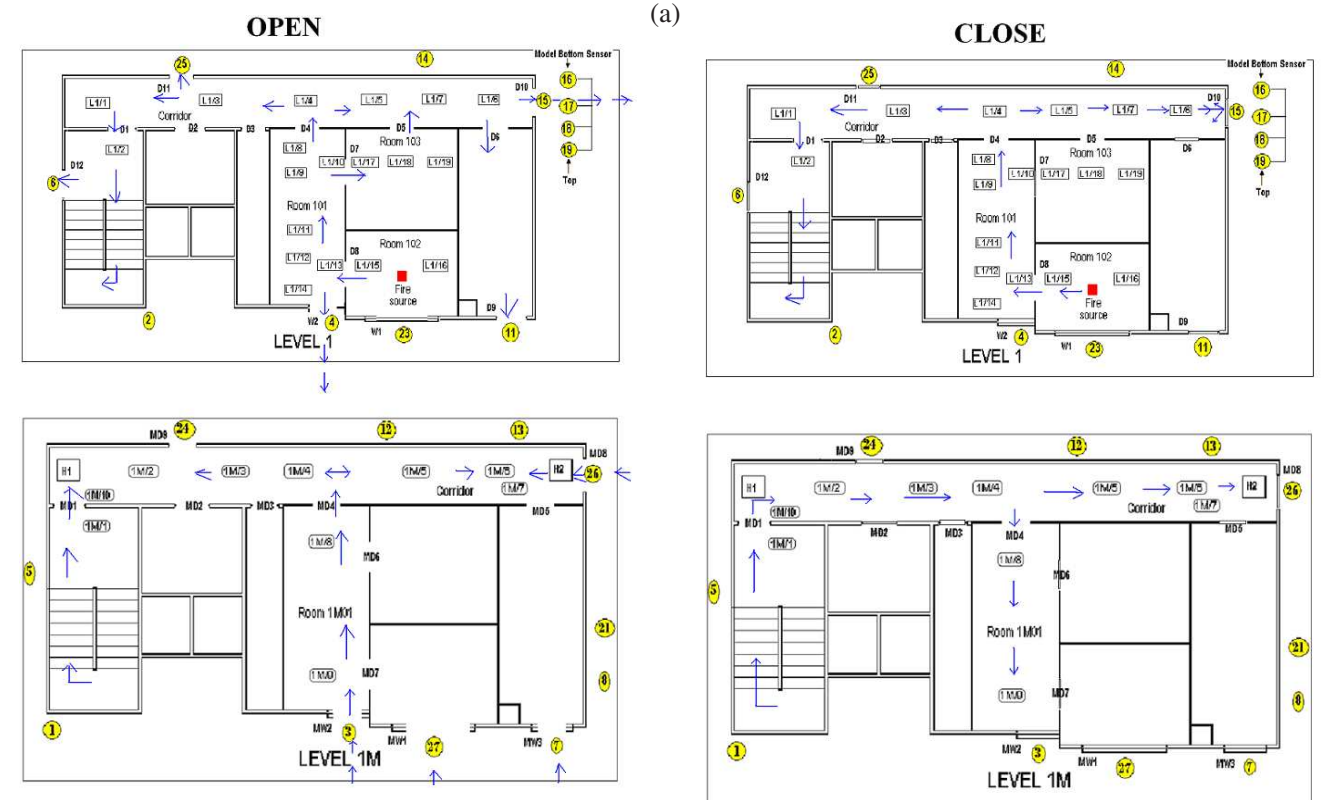

(b)

(c)

Figure 1. The experimental setup: (a) Image of the multi-compartment structure used for the experiment; (b) the flow path in the OPEN configuration; and, (c) the flow path in the CLOSE configuration.

Keywords: chemical tracer, concentration, statistics, multi-compartment 


\section{INTRODUCTION}

Detection of hazardous tracer releases is of great significance for many fields of science and technology spanning from technological and environmental monitoring to defence and security systems (see Lien et al. (2010), Dekker and Skvortsov (2009) and references therein). This topic is still an area of active research and there is a vast amount of literature devoted to this subject (see Farina et al. (2013), Rajasegarar et al. (2013), Mendis et al. (2012) and references therein).

In many practical settings, tracer particles (i.e., aerosol or gas molecules) are dispersed in confined structures (buildings, ships, tunnels, etc.) and this creates additional complexity for the underlying tracer field (e.g., stagnation zones, blockage, reconnection). Understanding this complexity (often associated with the spatialtemporal properties of tracer fluctuations) is an important step for the development of the architecture of future observation systems (number of sensors, thresholds, their density, etc.).

Many powerful analytical and numerical methods have been proposed to model tracer transport in confined structures that take into account the stochastic nature of underlying turbulent flow (e.g., CFD, Lagrangian transport). Unfortunately, while these high fidelity models provide valuable insights into the statistics of tracer distribution inside the structure, they often require expert-level knowledge and advanced computational capability in order to produce even the very basic estimates. This makes it difficult (or even unfeasible) deploying these advanced models for the design and development of hazard monitoring systems since this process involves continuous evaluation of numerous what-if scenarios and optimisation studies. This deficiency necessitates the development of simplified (but still scientifically consistent) models of tracer transport in confined environments that can easily be implemented and calibrated with a limited amount of experimental data. Revealing examples of such approaches are the compartment-based approach (Chen (2009)) and scaling models (Bakunin (2008), Skvortsov et al. (2013), Skvortsov et al. (2012)).

The presented study continues the same line of research by including statistical properties of tracer distribution in a confined environment. More specifically, we present a simple model of tracer fluctuations that captures their spatial variability induced by a multi-compartment structure. Some parameters of the model have been estimated with the results of a water tank experiment.

\section{TheORETICAL FRAMEWORK}

Some insight into tracer distribution inside the multi-compartment structure can be deduced based on the effective-media arguments (ben Avraham and Havlin (2000), Torquato (2002)). In the open space, the tracer concentration is governed by the diffusion equation (which is essentially a conservation law for tracer quantity), which in the steady state reduces to the Laplace equation (Csanady (1973)):

$$
D_{0} \Delta \theta=Q_{0} \delta(x-X, y-Y, z-Z) .
$$

Here $D_{0}$ is the diffusion coefficient of tracer in the open environment, $\Delta$ is the Laplace operator, $\theta$ is the tracer concentration, $\delta$ is the Dirac delta function, $Q_{0}$ is the release-rate of the tracer source, and $X, Y, Z$ are the coordinates of the source in a three-dimensional Cartesian coordinate system.

The solution of Eq. (1) reads

$$
\theta=\frac{Q_{0}}{4 \pi D_{0} R^{\nu}},
$$

where $\nu=2$, and $R$ is the distance between the tracer source and an observation point inside the structure: $R^{2}=(x-X)^{2}+(y-Y)^{2}+(z-Z)^{2}$.

The effective-media approach (ben Avraham and Havlin (2000), Torquato (2002)) eventually replaces the complex structure with some effective media with the same 'averaged' transport properties. More specifically, it states that after applying volume/ ensemble averaging to Eq. (1) it can be reduced to a similar equation, but for the mean concentration

$$
D \Delta\langle\theta\rangle=Q_{0} \delta(x-X, y-Y, z-Z),
$$

where $D$ is the so-called effective diffusivity that accounts for such a homogenisation, $\langle\theta\rangle$ is the time/ ensemble averaged tracer concentration. The new effective diffusivity $D$ is related to 'unobstructed' diffusivity $D_{0}$ of (1) via the formula $D=\epsilon D_{0}$. The scaling parameter $0 \leq \epsilon \leq 1$, known as tortuosity (Torquato (2002)), describes the 'aggregated' effect of the structure, the shape of compartments, and their connectivity (Torquato 
(2002)) and can be calculated numerically or, sometimes, analytically (Berezhkovskii et al. (2003), Torres et al. (2007)).

According to Eq.(3), a decrease of the effective diffusivity of tracer due to the presence of obstacles has the same effect as an appropriate increase of source release-rate (i.e., $Q=Q_{0} / \epsilon$ ), with unchanged diffusivity in (3) (i.e., $D=D_{0}$ ), where parameters $D_{0}, Q_{0}$ correspond to their values in an unobstructed space; see (1). We arrive at an important conclusion that within the effective media approach the effect of the structure can be approximately incorporated in the solution (2) with a simple renormalisation of the source release-rate $Q=Q_{0} / \epsilon$. In other words, it is reasonable to expect that for the mean (i.e., volume/ ensemble averaged) tracer concentration measured inside the multi-compartment structure one should still observe the scaling law $\langle\theta\rangle \propto R^{-\nu}$ with the exponent $\nu \approx 2$. It is worth noting that the case $\nu=0$ corresponds to a trivial scaling law $(\langle\theta\rangle=$ constant). We present both values of $\nu$ (namely, $\nu=2$ and $\nu=0$ ) for comparison.

Unfortunately, such simple reasoning is not applicable to the higher statistical moments of tracer distribution, since there are no simple conservation laws for quantities $\left\langle\theta^{n}\right\rangle$ (for $n>1$ ) that would allow us to write a closed equation similar to (3) (see Csanady (1973), Bakunin (2008), Celani et al. (2007) and references therein). Nevertheless, by employing the self-similarity reasoning within the effective media framework one may expect that a similar scaling law still holds: $\left\langle\theta^{n}\right\rangle \propto R^{-\mu_{n}}$, with some unknown exponents $\mu_{n}=$ const. For practical application the most important parameter from the set $\left\{\mu_{n}\right\}$ is the second exponent $\mu_{2}$ since it describes the distribution of tracer fluctuations inside the structure: $\sigma_{\theta}^{2}=\left\langle\theta^{2}\right\rangle-\langle\theta\rangle^{2}$. It is noteworthy that some bounds for the expected values for parameter $\mu_{2}$ can be deduced from the known properties of tracer plumes in obstructed canopies for which the asymptotes for ratio $i=\sigma_{\theta} /\langle\theta\rangle$ have been reported, namely, $i \rightarrow \infty$ near the boundaries of the plume and $i \rightarrow 0$ far downstream from the tracer source (Csanady (1973), Celani et al. (2007), Skvortsov et al. (2008)). Employing these results can often help in a qualitative interpretation of tracer statistics inside the complex structure.

\section{EXPERIMENTAL DATASET}

Experimental data described in this paper were obtained using a water tank experiment. A multiplecompartment acrylic model, constructed as a 1/15th scale model of a real building, was placed in a water tank filled with fresh water. A coloured saline solution with known density, released from a single point inside one compartment, was used to simulate a chemical tracer (Fig. 1(a)). Advection was provided along the length of the water-tank by water-pumps located on the upstream and downstream edges. The transport and dispersion of the tracer was then measured using conductivity sensors located at discrete locations inside the building. A set of collinear sensors were positioned along the centreline of each compartment to measure the tracer concentration at various altitudes. Doors of different compartments could be left in open or closed positions to make the plume traverse different paths through the multi-compartment structure. Flow paths corresponding to two such configurations, denoted as OPEN and CLOSE, are shown in Figures 1(b) and 1(c), respectively. More details of the experimental setup are available in Skvortsov et al. (2013).

As we can see from Figure 2, at each location, the signal generated by sensor tracer can be characterised by a number of parameters such as 'arrival time', 'saturation limit' and 'fluctuation level'(intensity of tracer fluctuations). The statistics of arrival times and saturation limits have been previously reported (Skvortsov et al. (2013)). Our aim in this paper is to investigate tracer fluctuations in different locations inside a multicompartment structure.

\section{RESUlts AND Discussion}

In this section, we present some preliminary results obtained by processing a small fraction of the data.

Time series in Figure 2 corresponding to OPEN and CLOSE configurations show the measured concentration at some sensors. Concentrations observed at some sensors under the OPEN and CLOSE configurations radically differ from one another while observed concentrations at some other sensors are fairly independent of the configuration.

Because the opening or closing of some doors of the multi-compartment structure change the plume flow paths, the 'walking distances' $L$ from the source to some sensors also change from one configuration to the other while the Eucidean distances $R$ remain unchanged. Figure 3(a) shows the relationship between $R$ and $L$ in the OPEN configuration while Figure 4(a) shows the corresponding relationship in the CLOSE configuration.

Figures 3(b) and (c) show mean concentration $\langle\theta\rangle$ and the square root of concentration variance, $\sigma_{\theta}$, as a function of $R$ in the OPEN configuration while $\sigma_{\theta}$ as a function of $\langle\theta\rangle$ is shown in Figure 3(d); values of $\langle\theta\rangle$ 
S. Karunasekera et al., Statistics of chemical tracer concentration in a multi-compartment structure

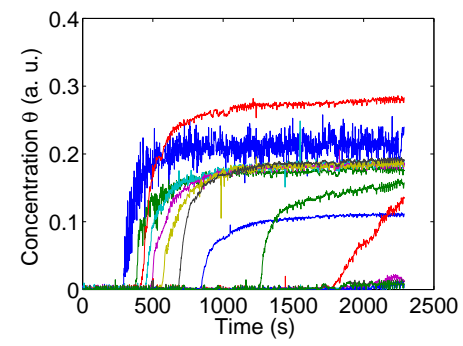

(a)

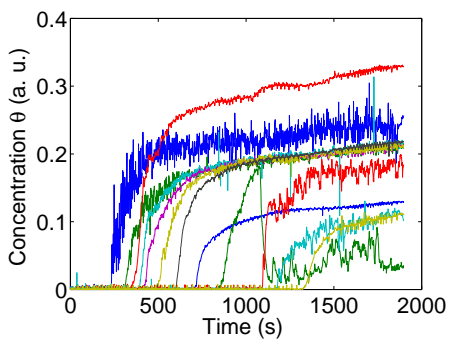

(b)

Figure 2. Concentration $\theta$ time series measured at some of the sensors in: (a) OPEN; and, (b) CLOSE configurations.

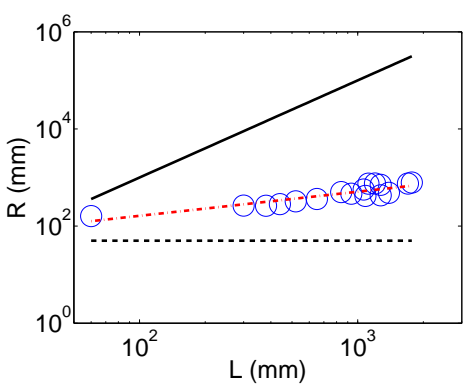

(a)

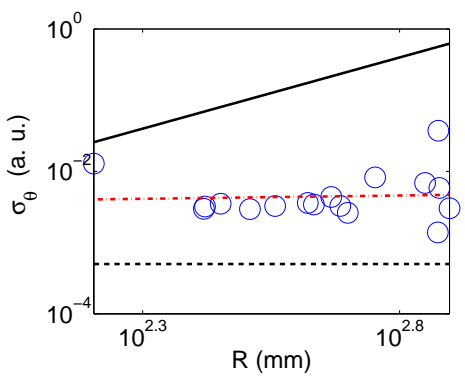

(c)

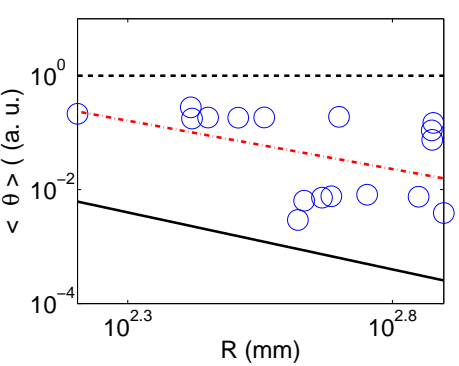

(b)

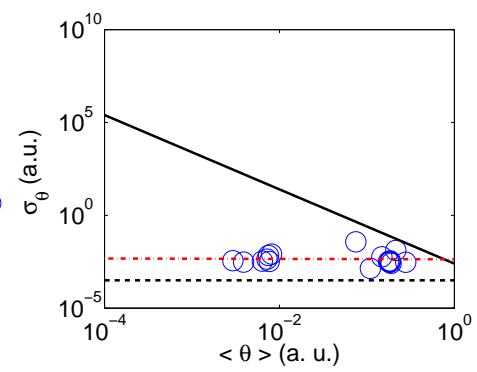

(d)

Figure 3. Plots of: (a) Euclidean distance $R$ vs. 'walking distance' $L$; power law $R \propto L^{\beta}$ with $\beta=2(-)$, $\beta=0$ (---), $\beta=0.5$ (......); (b) Mean concentration $\langle\theta\rangle$ vs. $R$; power law $\langle\theta\rangle \propto R^{-\nu}$ with $\nu=2(-)$, $\nu=0(--), \nu=1.7$ ( $\cdots \cdots . .$.$) ; (c) \sigma_{\theta}$ vs. $R$; power law $\sigma_{\theta} \propto R^{\mu}$ with $\mu=2(-), \mu=0(--), \mu=0.2$

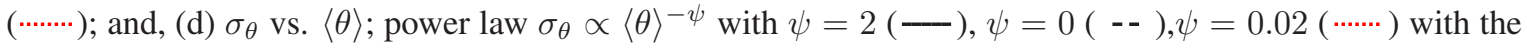
multi-compartment structure in the OPEN configuration. In all plots, symbols $(\bigcirc)$ denote data points and the dotted red lines (........) show the least squares fit lines. $\sigma_{\theta}$ is the square root of concentration variance.

and $\sigma_{\theta}$ were computed using concentration data extracted from a narrow temporal window located at the end of each concentration time series.

Because the theory presented above suggests power law relationships between these quantities, we plot the least squares fit lines and also some straight lines that represent some hypothetical power law relationships on these figures to provide a visual guidance to the reader. The corresponding plots obtained with the model structure in the CLOSE configuration are presented in Figure 4.

Next, in Figure 5, we present: (a) a typical concentration $\theta$ time series observed at a sensor, followed by; (b) the mean concentration $\langle\theta\rangle$; and (c) concentration variance $\sigma_{\theta}$ obtained using a narrow moving window; and, (d) intensity $i=\sigma_{\theta} /\langle\theta\rangle$. Intensity time series similar to that in Figure 5(d) were obtained for all sensors in both OPEN and CLOSE configurations. Figure 6 shows plots of the values of intensity $i$ extracted at three 


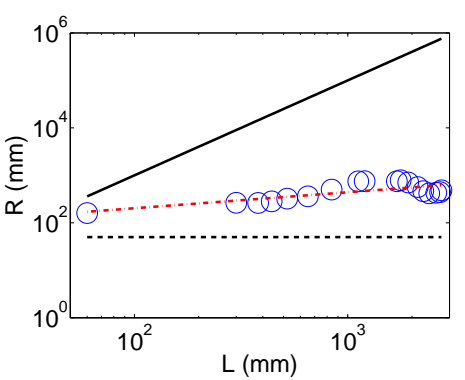

(a)

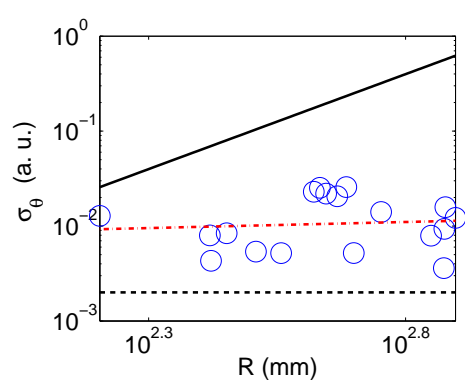

(c)

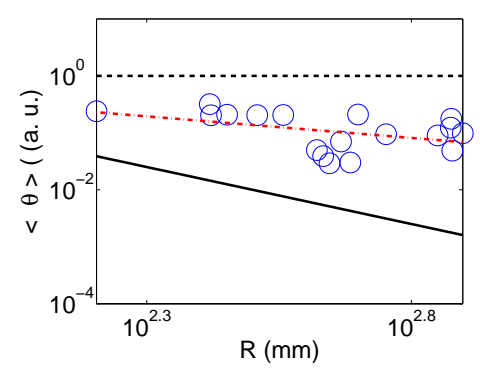

(b)

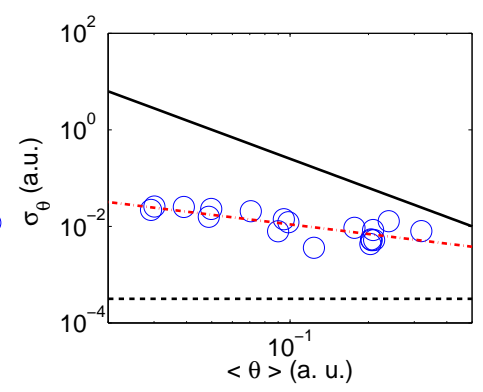

(d)

Figure 4. Plots of: (a) Euclidean distance $R$ vs. 'walking distance' $L$; power law $R \propto L^{\beta}$ with $\beta=2(-)$, $\beta=0$ (---), $\beta=0.34$ ( …...); (b) Mean concentration $\langle\theta\rangle$ vs. $R$; power law $\langle\theta\rangle \propto R^{-\nu}$ with $\nu=2(-)$, $\nu=0(--), \nu=0.76$ ( …...); (c) $\sigma_{\theta}$ vs. $R$; power law $\sigma_{\theta} \propto R^{\mu}$ with $\mu=2(-), \mu=0(--), \mu=0.13$ (…....); and, (d) $\sigma_{\theta}$ vs. $\langle\theta\rangle$; power law $\sigma_{\theta} \propto\langle\theta\rangle^{-\psi}$ with $\psi=2(-), \psi=0(---), \psi=1.32$ ( $\ldots \ldots \ldots$ ) $)$ with the multi-compartment structure in the CLOSE configuration. In all plots, symbols $(\bigcirc)$ denote data points and the dotted red lines (.......) show the least squares fit lines. $\sigma_{\theta}$ is the square root of concentration variance.

different times $\left(t=0(\bigcirc), t=T_{\max } / 2(\times)\right.$, and $\left.t=T_{\max }(\square)\right)$ from each such time series plotted against the Euclidean distance $R$ between the corresponding sensor and the source; here, $T_{\max }$ is the total time over which tracer measurements were made.

\section{CONCLUding REMARKS}

The framework presented above enables important decisions to be made about parameters of monitoring systems. For example, consider the design of a chemical sensor network to monitor hazardous releases in a multicompartment structure. In operational settings, the requirements for such a system are usually formulated in terms of $\langle\theta\rangle$ (level of hazardous concentration to be detected) and $T$ (time interval within which it should be detected to mitigate the impact of the release). Let us assume that we are trying to deploy a system of chemical sensors that are capable of producing a concentration measurement during the time interval $\tau$ with the detection threshold $\mu$. For a given environment and a location, the required number of sensors, $N$, is given by an estimate $N(T / \tau) P_{D} \geq 1$ where $P_{D}$ is the probability of detection defined as $P_{D}=\operatorname{Pr}(\theta \geq \mu), 1-F\left(\mu, i^{2} \mu\right)$ where $F\left(\mu, i^{2} \mu\right)$ is the cumulative distribution function of concentration fluctuations (for models of $F$ see Gunatilaka et al. (2012)); $i=\sigma_{c} /\langle\theta\rangle$ is fluctuation intensity (given by measurements presented in Fig. 6). From here, we can derive the following criterion $N>N_{*}$, where

$$
N_{*}=\tau /\left(P_{D} T\right) .
$$

In operational scenarios, we often have an additional constraint $T \mu<S=$ constant, where $S$ is the maximum toxic dose. Then Eq. 4 will define our optimisation condition, and can be used in 'what-if' analysis studies.

To conclude we list the main results of our study:

1. We implemented a model of tortuosity in application to our multi-component structure. This model relates the walking distance and the Euclidian distance to the tracer source. According to the results, the tortuosity can be introduced as $\epsilon=R / L \sim L^{\beta-1}$ where $\beta \sim 0.5$ (Fig. 3(a)). The concept of 
S. Karunasekera et al., Statistics of chemical tracer concentration in a multi-compartment structure

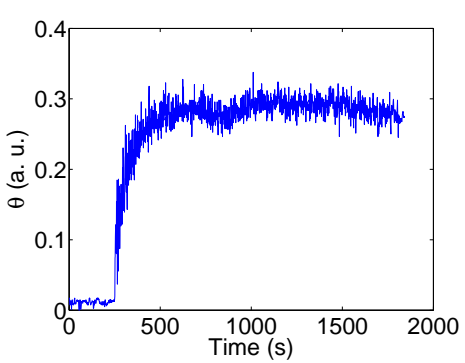

(a)

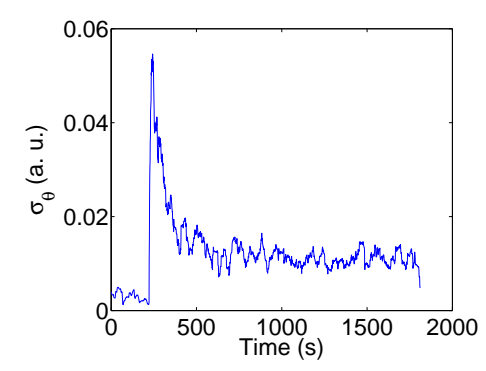

(c)

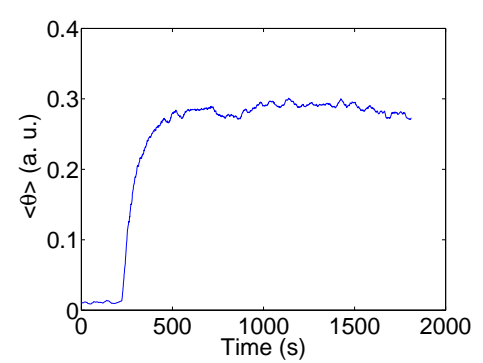

(b)

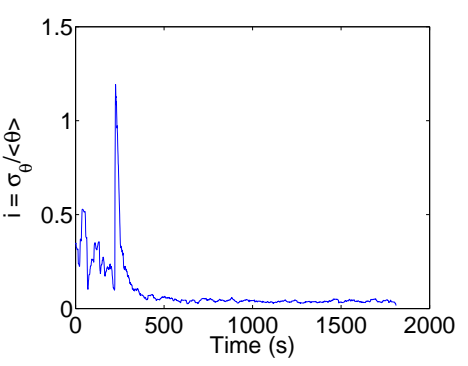

(d)

Figure 5. Plots of: (a) Concentration $\theta$; (b) Mean concentration $\langle\theta\rangle$; (c) $\sigma_{\theta}$; and, (d) Intensity $i=\sigma_{\theta} /\langle\theta\rangle$ vs. time for a typical sensor. $\sigma_{\theta}$ is the square root of concentration variance.

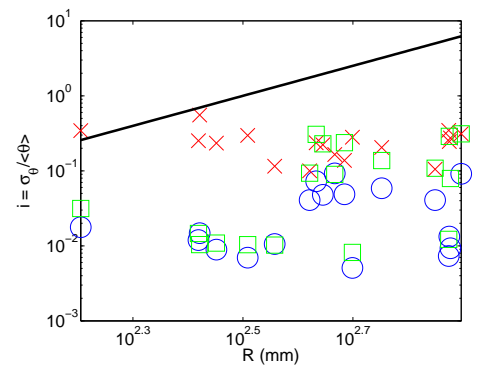

(a)

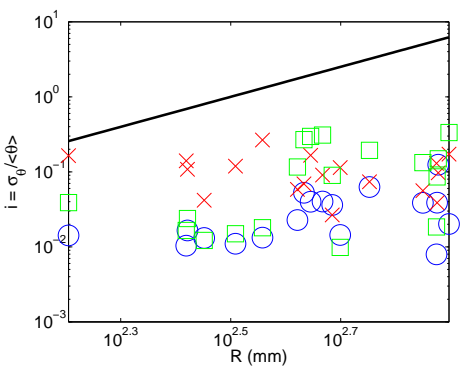

(b)

Figure 6. Intensity $i=\sigma_{\theta} /\langle\theta\rangle$ vs. Euclidean distance at $t=0(\bigcirc), t=T_{\max } / 2(\times)$, and $t=T_{\max }(\square)$ for a selected sensor in: (a) OPEN; and, (b) CLOSE configurations. $T_{\max }$ is the total time over which tracer measurements were made. Lines ( - ) show the power law $i \propto R^{\zeta}$ with $\zeta=2$.

tortuosity is important for the development of a unified framework for source backtracking algorithms in multi-compartment structures (since walking distances are unknown beforehand.)

2. For a number of illustrative scenarios we fit the experimental data profiles to the scaling laws. We found that for some scenarios the power-law fit performs reasonably well (Figs. 4(d)), while for others it is rather poor (Figs. 3(d)). The poor agreement of some experimental data profiles to power laws may be attributed to the measured concentrations never reliably reaching the saturation limit in our experimental settings (see Figure 2) whereas the theoretical value for the power law exponent is valid for the saturation limit. More definitive conclusions about quality of the power-law fit may be drawn after processing the entire dataset.

3. Relative fluctuation intensity $i=\sigma_{\theta} /\langle\theta\rangle$ is also approximately constant across the two structures. It has a value of around 0.01 at saturation limit. It does change during transition time (Fig. 6). 
Furthermore, as mentioned above, the preliminary results presented above are based on a subset (about 20\%) of data obtained in our experimental study. More comprehensive analysis will be reported in a separate publication.

We anticipate that the results presented in this study can be useful in evaluating networking systems for environmental monitoring and in data fusion work for source backtracking within multi-compartment structures.

\section{ACKNOWLEDGEMENT}

Authors like to thank B. Suendermann, G. Gamble, I. Burch, A. Douglas, and J. Robinson for their help with the experimental setup and data collection. Authors also like thank Ralph Gailis for his careful reading of the paper and valuable suggestions.

\section{REFERENCES}

Bakunin, O. G. (2008). Turbulence and diffusion: scaling versus equations. Springer, Berlin.

ben Avraham, D. and S. Havlin (2000). Diffusion and reaction in fractals and disordered systems. Cambridge University Press.

Berezhkovskii, A. M., V. Y. Zitserman, and S. Y. Shvartsman (2003). Effective diffusivity in periodic porous materials. J. Chem. Phys. 119, 6991-6993.

Celani, A., M. M. Afonso, and A. Mazzino (2007). Point-source scalar turbulence. Journal of Fluid Mechanics 583, 189-198.

Chen, Q. (2009). Ventilation performance prediction for buildings: A method overview and recent applications. Building and Environment 44, 848-858.

Csanady, G. T. (1973). Turbulent diffusion in the environment. D. Reidel Publishing Company, USA.

Dekker, A. and A. Skvortsov (2009). Topological issues in network of chemical sensors. In Proc. MODSIM2009.

Farina, A., L. Ortenzi, B. Ristic, and A. Skvortsov (2013). Integrated Sensor Systems and Data Fusion for Homeland Protection, Volume Academic Press Library in Signal Processing, Chapter 22. Elsvier.

Gunatilaka, A., A. Skvortsov, and R. Gailis (2012, July 9-12). High fidelity simulation of hazardous plume concentration time series based on models of turbulent dispersion. In 15th International Conference on Information Fusion, Singapore, pp. 1838 - 1845.

Lien, F. S., H. Ji, E. C. Yee, and B. Kournikakis (2010). Prediction of aerosol hazard arising from the opening of an anthrax letter in an open-office environment using computational fluid dynamics. Journal of Engineering Science \& Technology 5(3), 302-331.

Mendis, C., A. Skvortsov, A. Gunatilaka, and S. Karunasekera (2012). Performance of wireless chemical sensor network with dynamic collaboration. IEEE Sensors Journal 12(8), 2630-2637.

Rajasegarar, S., T. C. Havens, S. Karunasekera, C. Leckie, J. C. Bezdek, M. Jamriska, A. Gunatilaka, A. Skvortsov, and M. Palaniswami (2013). High resolution monitoring of atmospheric pollutants using system of low-cost sensors. IEEE Transactions on Geoscience \& Remote Sensing. In review.

Skvortsov, A., B. Suendermann, G. Gamble, M. Roberts, O. Ilaya, and D. Pitaliadda (2012). Tracer dispersion in a multi-compartment structure. In Proc. 18th Australasian Fluid Mech. Conf., Launceston, Australia.

Skvortsov, A., B. Suendermann, G. Gamble, M. Roberts, O. Ilaya, and D. Pitaliadda (2013). Scaling laws of tracer dispersion in a multi-compartment structure. Experimental Thermal and Fluid Science 45, 110-116.

Skvortsov, A. T., P. D. Dawson, M. D. Roberts, and R. M. Gailis (2008). Modelling of flow and tracer dispersion over complex urban terrain in the atmospheric boundary layer. In WSEAS Transactions on Fluid Mechanics.

Torquato, S. (2002). Heterogeneous materials. Springer.

Torres, F., M. Gauthier, J. .-F. Mercier, and G. W. Slater (2007). Diffusion of small particles in a network of square cavities: Exact numerical results. Physics in Canada 61(3). 